\title{
Primary Care Medication Safety Surveillance with Integrated Primary and Secondary Care Electronic Health Records: A Cross-Sectional Study
}

\author{
Artur Akbarov $^{1} \cdot$ Evangelos Kontopantelis $^{1,2} \cdot$ Matthew Sperrin $^{1} \cdot$ Susan J. Stocks ${ }^{3}$. \\ Richard Williams $^{1,3} \cdot$ Sarah Rodgers $^{4}$ - Anthony Avery ${ }^{4} \cdot$ Iain Buchan $^{1,3}$. \\ Darren M. Ashcroft ${ }^{3,5}$
}

Published online: 23 June 2015

(c) The Author(s) 2015. This article is published with open access at Springerlink.com

\begin{abstract}
Introduction The extent of preventable medication-related hospital admissions and medication-related issues in primary care is significant enough to justify developing decision support systems for medication safety surveillance. The prerequisite for such systems is defining a relevant set of medication safety-related indicators and understanding the influence of both patient and general practice characteristics on medication prescribing and monitoring.

Objective The aim of the study was to investigate the feasibility of linked primary and secondary care electronic health record data for surveillance of medication safety,
\end{abstract}

Electronic supplementary material The online version of this article (doi:10.1007/s40264-015-0304-x) contains supplementary material, which is available to authorized users.

Darren M. Ashcroft

darren.ashcroft@mancheser.ac.uk

Artur Akbarov

artur.akbarov@manchester.ac.uk

1 Centre for Health Informatics, Institute of Population Health, University of Manchester, Vaughan House, Portsmouth St, Manchester M13 9GB, UK

2 NIHR School for Primary Care Research, University of Manchester, Oxford Road, Manchester M13 9PL, UK

3 NIHR Greater Manchester Primary Care Patient Safety Translational Research Centre, University of Manchester, Oxford Road, Manchester M13 9PL, UK

4 Division of Primary Care, School of Medicine, University of Nottingham, Queen's Medical Centre, Nottingham NG7 2UH, UK

5 Centre for Pharmacoepidemiology and Drug Safety, Manchester Pharmacy School, University of Manchester, Oxford Road, Manchester M13 9PT, UK examining not only prescribing but also monitoring, and associations with patient- and general practice-level characteristics.

Methods A cross-sectional study was conducted using linked records of patients served by one hospital and over 50 general practices in Salford, UK. Statistical analysis consisted of mixed-effects logistic models, relating prescribing safety indicators to potential determinants.

Results The overall prevalence (proportion of patients with at least one medication safety hazard) was $5.45 \%$ for prescribing indicators and $7.65 \%$ for monitoring indicators. Older patients and those on multiple medications were at higher risk of prescribing hazards, but at lower risk of missed monitoring. The odds of missed monitoring among all patients were $25 \%$ less for males, $50 \%$ less for patients in practices that provide general practitioner training, and threefold higher in practices serving the most deprived compared with the least deprived areas. Practices with more prescribing hazards did not tend to show more monitoring issues.

Conclusions Systematic collection, collation, and analysis of linked primary and secondary care records produce plausible and useful information about medication safety for a health system. Medication safety surveillance systems should pay close attention to patient age and polypharmacy

\section{Key Points}

Linked primary and secondary health care data are important for comprehensive medication safety surveillance.

Medication prescribing and monitoring should be treated as different statistical processes. 
with respect to both prescribing and monitoring failures; treat prescribing and monitoring as different statistical processes, rather than a combined measure of prescribing safety; and audit the socio-economic equity of missed monitoring.

\section{Introduction}

Prescribing and monitoring of drug therapies are important areas for clinical quality improvement. Among drug-related hospital admissions, around $59 \%$ are thought to be preventable; this represents approximately $4 \%$ of all hospital admissions $[1,2]$. The majority of these admissions involved antiplatelet drugs, diuretics, non-steroidal antiinflammatory drugs, or anticoagulants [2].

There have been numerous studies from different countries that addressed the issue of inappropriate prescribing. Different prevalence rates have been reported: $13.9 \%$ [3], $14.6 \%$ [4], $22 \%$ [5], and $31.9 \%$ [6]. Most studies focused on prescribing indicators and/or elderly patients; medication monitoring received much less attention. The reported extent of inappropriate medication therapies clearly warrants systematic intervention [3, 7, 8]. A pharmacist-led intervention, Pharmacist-led ITbased iNtervention with simple feedback in reducing rates of Clinically important ERrors (PINCER) [8], using UK primary care data showed that prescribing errors could be reduced through surveillance and pharmacy-led feedback to general practitioners. Data that might be used to support safer therapy in real time exist in electronic health records; however, most health care data is spread across multiple, unlinked databases. In this study, we used linked secondary and primary care records, an early instance of the global trend in electronic health record development, from the population of Salford, UK. Salford, a city and one of the ten metropolitan boroughs of Greater Manchester in the North West of England, is a relatively deprived population of approximately 234,000 [9] (usual residents) served by a single hospital and over 50 general practices.

This study aimed to investigate the feasibility of wideranging medication safety surveillance using a linked database. Future quality improvement interventions based on such surveillance will require detailed, local understanding of factors that drive medication safety. Therefore, the objectives of this study included assessing the prevalence of 22 medication safety indicators, investigating associations with patient and practice characteristics, and investigating variation between general practices.

\section{Methods}

\subsection{Data Source}

Over the past 20 years, the National Health Service (NHS) in Salford, UK, has been working toward integrating hospital- and community-based services, starting with shared information about patients-initially a series of chronic disease registers, later merging into the Salford Integrated Record (SIR). SIR now draws data on a daily basis from primary care practices and the hospital at the Salford Royal NHS Foundation Trust. The integrated record is available to clinicians via the hospital's electronic patient record system for individual patients, for example, to check primary care records of allergy in a patient presenting at the emergency department. SIR is also available in linked, anonymised form for research and service development purposes.

\subsection{Indicator Definition}

We define an indicator of medication safety as the proportion of patients for whom an appropriate medication policy has not been followed, or not recorded as such in the electronic health record, in a given at-risk group/context. The denominator consists of all patients for whom the medication policy should be followed; the numerator consists of the subgroup of patients in the denominator for whom the medication policy has not been followed. The appropriateness of such policies were derived from bestpractice guidelines or determined via consensus methods by a panel of experts $[10,11]$. We considered 18 prescribing and four monitoring indicators. These indicators are described in Table 1 and have been reported by Avery et al. [10] and Spencer et al. [11]. They were developed through an extensive literature review and a rigorous consensus process and were deemed to be most likely associated with preventable drug-related morbidity when applied to electronic health records in primary care in the UK $[2,10,11]$. Some other indicator sets include the Beers criteria [12], Assessing Care Of Vulnerable Elders (ACOVE) [13], and Screening Tool of Older Persons' potentially inappropriate Prescriptions (STOPP) $[14,15]$ to name a few. We had to exclude some of the indicators from the sets reported in $[8,10,11]$ from our analysis because SIR filters mental and sexual health records to preserve patient privacy.

All indicators were defined with respect to a given audit date-reference date. For the primary analysis, we chose 1 April 2012 as the reference date because it aligns with the start of the 2012/2013 NHS financial year. For the analyses 
Table 1 Indicators of unsafe medication practice

\begin{tabular}{|c|c|c|c|c|}
\hline & ID & Potential hazard/at-risk patient group (1 April 2012) & Prevalence & ICC \\
\hline \multirow[t]{18}{*}{ Prescribing } & P1 & $\begin{array}{l}\text { Prescribed aspirin or clopidogrel/patients not prescribed gastro-protection who have a } \\
\text { history of peptic ulcer }\end{array}$ & $12.31 \%(216 / 1755)$ & 0.004 \\
\hline & $\mathrm{P} 2$ & Prescribed aspirin/patients prescribed warfarin without co-prescription of gastro-protection & $4.52 \%(77 / 1705)$ & 0 \\
\hline & P3 & Prescribed an NSAID/patients prescribed warfarin & $5.20 \%(159 / 3060)$ & 0.006 \\
\hline & $\mathrm{P} 4$ & $\begin{array}{l}\text { Prescribed an NSAID/patients not prescribed gastro-protection who have a history of } \\
\text { peptic ulcer }\end{array}$ & $5.53 \%(97 / 1755)$ & 0 \\
\hline & P5 & Prescribed an NSAID/patients aged 65 or older without prescription of gastro-protection & $6.20 \%(1563 / 25,228)$ & 0.023 \\
\hline & P6 & Prescribed an NSAID/patients with diagnosis of CKD $(3 \mathrm{~b}, 4,5)$ or with latest eGFR $<45$ & $3.03 \%(108 / 3570)$ & 0.013 \\
\hline & P7 & $\begin{array}{l}\text { Prescribed an NSAID/patients with diagnosis of CKD }(3 \mathrm{~b}, 4,5) \text { or with latest eGFR }<45 \\
\text { who have been prescribed an ACEi }\end{array}$ & $4.01 \%(70 / 1746)$ & 0.021 \\
\hline & P8 & $\begin{array}{l}\text { Prescribed an NSAID/patients with diagnosis of CKD }(3 \mathrm{~b}, 4,5) \text { or with latest eGFR }<45 \\
\text { who are prescribed an ACEi and a loop diuretic }\end{array}$ & $3.99 \%(27 / 677)$ & 0 \\
\hline & P9 & Prescribed metformin/patients with diagnosis of $\operatorname{CKD}(4,5)$ or with latest eGFR $<30$ & $3.71 \%(46 / 1241)$ & 0 \\
\hline & $\mathrm{P} 10$ & $\begin{array}{l}\text { Prescribed digoxin at a daily dose of }>125 / \text { patients with a diagnosis of CKD }(3 \mathrm{~b}, 4,5) \text { or } \\
\text { with latest eGFR }<45\end{array}$ & $0.31 \%(11 / 3570)$ & 0 \\
\hline & $\mathrm{P} 11$ & Prescribed an NSAID/patients with heart failure & $3.17 \%(94 / 2964)$ & 0.003 \\
\hline & $\mathrm{P} 12$ & Prescribed glitazone/patients with heart failure & $1.89 \%(56 / 2964)$ & 0.001 \\
\hline & $\mathrm{P} 13$ & Prescribed $\beta$-blockers/patients with unresolved asthma & $4.73 \%(1258 / 26,607)$ & 0.002 \\
\hline & P14 & $\begin{array}{l}\text { Not prescribed inhaled corticosteroid/patients with asthma prescribed a long-acting } \\
\beta \text {-agonist }\end{array}$ & $1.65 \%(79 / 4796)$ & 0.007 \\
\hline & P15 & $\begin{array}{l}\text { Prescribed combined hormonal contraceptive/women with history of venous or arterial } \\
\text { thrombosis }\end{array}$ & $0.13 \%(14 / 10,889)$ & 0 \\
\hline & $\mathrm{P} 16$ & Prescribed combined hormonal contraceptive/women with a body mass index of $\geq 40$ & $2.78 \%(109 / 3927)$ & 0.012 \\
\hline & $\mathrm{P} 17$ & Prescribed combined hormonal contraceptive/women aged $\geq 35$ who are current smokers & $1.12 \%(171 / 15,286)$ & 0.004 \\
\hline & P18 & Prescribed oral or transdermal oestrogens/women with a history of breast cancer & $1.33 \%(23 / 1724)$ & 0.002 \\
\hline \multirow[t]{4}{*}{ Monitoring } & M1 & $\begin{array}{l}\text { Missing renal function and electrolytes in the past } 15 \text { months/patients aged } \geq 75 \text { on a long- } \\
\text { term ACEi or loop diuretic }\end{array}$ & $5.06 \%(411 / 8123)$ & 0.037 \\
\hline & M2 & $\begin{array}{l}\text { Missing full blood count or liver function test in the past } 3 \text { months/patients receiving } \\
\text { repeat methotrexate }\end{array}$ & $6.74 \%(31 / 460)$ & 0.079 \\
\hline & M3 & $\begin{array}{l}\text { Missing International Normalised Ratio test in the past } 3 \text { months/patients receiving repeat } \\
\text { warfarin }\end{array}$ & $9.95 \%(244 / 2452)$ & 0.213 \\
\hline & M4 & Missing thyroid function test in the past 6 months/patients receiving repeat amiodarone & $38.01 \%(103 / 271)$ & 0.047 \\
\hline
\end{tabular}

$\overline{A C E i}$ angiotensin converting enzyme inhibitor, $C K D$ chronic kidney disease, $e G F R$ estimated glomerular filtration rate, $I C C$ intra-class correlation coefficient (proportion of the total variation explained by the variation between practices), NSAID non-steroidal anti-inflammatory drug (only non-selective considered)

using alternative reference dates, please see the electronic supplementary material (online resource 1).

\subsection{Covariates}

Patient-level covariates considered in this study were age, gender, patient's overall Index of Multiple Deprivation (IMD) [16] for the year 2010, and polypharmacy. The IMD is a weighted combination of the following seven distinct deprivation domains (weight): income deprivation $(22.5 \%)$, employment deprivation $(22.5 \%)$, health deprivation and disability (13.5\%), education skills and training deprivation $(13.5 \%)$, barriers to housing and services $(9.3 \%)$, living environment deprivation $(9.3 \%)$, and crime
$(9.3 \%)$. It is measured at the level of small areas, lower layer super output areas (LSOAs), of approximately equal size - on average 1500 people. Lower values of IMD are associated with less deprivation. Polypharmacy, the number of simultaneous repeat prescriptions at the reference date, was calculated as the number of drugs with at least two electronic records of prescription on different days within the last 12 months; different doses and switching between different brands of the same medication were not counted.

Practice-level covariates were number of patients per general practice (list size), clinical software used (Vision vs. EMIS), whether a practice is involved in providing general practitioner training or not, average age of the 
patient population, and practice level deprivation. The latter was calculated as the average of individual patient IMD values.

\subsection{Statistical Methods}

The main statistical analysis consisted of mixed-effects logistic regression modelling, where the dependent variable was the patient-level medication policy outcome $(0=$ followed, $1=$ not followed $)$. Two separate models were fitted using the lme4 package in R [17, 18]: one for all prescribing indicators and one for all monitoring indicators. This was considered to give the best balance between an overwhelming number of underpowered models (if each indicator was considered separately) and excessive heterogeneity (if monitoring and prescribing indicators were considered together). For each model, the fixed effects were given by the abovementioned covariates, whereas the random effects were given by the following grouping factors: indicator label, general practice label, and patient label.

The variation between indicators and practices was examined before and after adjusting for the covariates. The minimal adequate models for the adjustment were selected on the basis of the minimum Akaike Information Criterion (AIC) [19], through an exhaustive search over all covariate combinations. For the analysis of indicator-specific random effects on covariate coefficients, please see the electronic supplementary material.

$R^{2}$, the percentage of variation explained by a model, is reported in two forms, as recommended by Nakagawa and Schielzeth [20]: marginal, the variation explained by fixed effects only (covariates), and conditional, the variation explained by covariates as well as the variation explained by differences between indicators, practices, and patients (random effects).

The relative variation between practices within each indicator was quantified using the intra-class correlation coefficient (ICC) [21], which measures the proportion of variation explained by the variation between practices in indicator-specific models without covariates.

Results are presented as main effects with a $95 \%$ confidence interval.

\section{Results}

We considered 52 general practices. The median list size was 3492 (range 797-16,710), the average age of patients on the list was 38.89 years (standard deviation 7.96), and the average deprivation of the list was 37.03 (standard deviation 10.19). There were eight training practices, 39 practices using Vision software and 13 using EMIS.
The summary of patient-level characteristics is given in Table 2. The total adult population was 205,519-all patients 18 years old or older affiliated with the 52 general practices considered in this study. This number also includes university students (not usual residents) in Salford. As an example, we can note from Table 2 that patients with no monitoring hazards are older on average (79.03) than patients with monitoring hazards (75.72). Conversely, the average age of patients with prescribing hazards (65.33) is older than the average age of patients with no prescribing hazards (56.92).

\subsection{Prevalence and Variation Between Practices for Each Indicator}

Table 1 shows the prevalence and the ICC for each indicator. Prevalence ranged from 0.13 to $12.31 \%$, apart from an outlier, M4 (patients on amiodarone without recent thyroid function test), which was $38.01 \%$. The overall prevalence (proportion of patients with at least one medication safety hazard) was $5.45 \%$ for prescribing indicators and $7.65 \%$ for monitoring indicators $(6.77 \%$ excluding M4).

The ICC values for monitoring indicators were notably higher than those for prescribing indicators. This suggests that, for monitoring indicators, the variation between practices accounts for a higher percentage of the total variation. The highest ICC, by far, was for M3 (patients on warfarin without a recent International Normalised Ratio test).

\subsection{Covariate Effects}

Table 3 shows estimated odds ratios for univariate and adjusted models. The prevalence values for each variable band reported in the table represent the proportion of patients with at least one missed monitoring or hazardous prescribing. The following important results stand out. Age and polypharmacy displayed opposite effects for prescribing and monitoring indicators: higher age and polypharmacy were associated with increased odds of a prescribing hazard, but decreased odds of potential monitoring failures. Only age and polypharmacy appear in both adjusted models. So, age and polypharmacy are important factors for both monitoring and prescribing indicators. Although, there is a clear relationship between age and polypharmacy, there was no statistically significant interaction between these two covariates. The odds of missing a medication monitoring event for male patients were $25 \%$ less than for female patients. The odds of missing a medication monitoring event for patients in training practices were $50 \%$ less than for patients in non-training practices. Practice-level deprivation was important only for 
Table 2 Adult population (18 years old or older) characteristics by medication hazard types; average (standard deviation)

\begin{tabular}{|c|c|c|c|c|c|c|c|}
\hline & \multirow[t]{2}{*}{ All } & \multicolumn{2}{|l|}{ Monitoring } & \multicolumn{2}{|l|}{ Prescribing } & \multicolumn{2}{|c|}{ Monitoring and prescribing } \\
\hline & & $\begin{array}{l}\text { No } \\
\text { hazards* }\end{array}$ & $\begin{array}{l}\text { At least } \\
\text { one hazard }\end{array}$ & $\begin{array}{l}\text { No } \\
\text { hazards* }\end{array}$ & $\begin{array}{l}\text { At least } \\
\text { one hazard }\end{array}$ & $\begin{array}{l}\text { No } \\
\text { hazards* }\end{array}$ & $\begin{array}{l}\text { At least one combination } \\
\text { of monitoring and } \\
\text { prescribing hazard }\end{array}$ \\
\hline Number & 205,519 & 9353 & 775 & 66,537 & 3833 & 7338 & 71 \\
\hline Age & $46.21(19.3)$ & $79.03(9.53)$ & $75.72(13.95)$ & $56.92(20.19)$ & $65.33(16.03)$ & $79.42(9.23)$ & $74.68(13.46)$ \\
\hline IMD & $35.17(18.83)$ & $33.56(18.47)$ & $36.65(18.97)$ & $35.22(18.82)$ & $34.47(18.97)$ & $33.64(18.44)$ & $36.68(20.65)$ \\
\hline Polypharmacy & $2.1(3.5)$ & $8.63(4.33)$ & $7.59(4.27)$ & $3.73(4.28)$ & $7.12(5.14)$ & $8.42(4.31)$ & $8.55(4.16)$ \\
\hline Males (\%) & 51 & 44 & 43 & 39 & 44 & 44 & 52 \\
\hline
\end{tabular}

IMD Index of Multiple Deprivation

* The "no hazards" columns are not the denominator values; they represent the number of patients who were in the denominator but not in the numerator of any of the indicators

monitoring indicators. The main difference was between least deprived practices and most deprived practices, where the odds of potential monitoring failure in the latter were threefold higher. Patient-level deprivation was found to be important only for prescribing indicators. The only significant difference was between least deprived and most deprived patients, where the odds of a hazardous prescribing event for the latter were $15 \%$ less. This result should be treated with caution as there were no significant differences between different patient deprivation groups in the univariate model. Additional analyses showed that the estimates for patient-level deprivation were mostly affected by adjusting for polypharmacy. The odds of having a prescribing hazard apparent in Vision-derived data appeared to be $20 \%$ less than in EMIS-derived data. However, the difference was not statistically significant at a $5 \%$ significance level.

The marginal $R^{2}$, which measures the variation explained by covariates in the adjusted models, was $11.1 \%$ for prescribing indicators and $7.6 \%$ for monitoring. Similar results were reported in Guthrie et al. [3], where adjusted models for high-risk prescribing explained around 8-9 \% of the total variation.

The conditional $R^{2}$ was $49.8 \%$ for prescribing indicators and $43.6 \%$ for monitoring. That is, $49.8 \%$ of the variation for prescribing indicators was explained by both fixed and random effects of the adjusted model; similarly for monitoring indicators.

\subsection{Variation Between Practices}

The variation between practices, measured by the variance of random effects for practice labels, before adjusting for covariates was 0.65 and 0.14 (on $\log$ odds scale) for monitoring and prescribing indicators, respectively. In the adjusted models, this variation reduced to 0.44 and 0.12 , respectively.

The variation between practices in the adjusted models is depicted in Fig. 1 [22]. It shows, for each practice, practice-specific effects not captured by covariates considered in this study (the effects are on a log odds scale). Practices with values below zero are less likely to be associated with medication safety indicators than practices with values above zero. The $95 \%$ prediction intervals reflect the uncertainty about the predicted random effects. The most interesting thing to note from Fig. 1 is that high prevalence for prescribing safety indicators does not necessarily go hand in hand with high prevalence for medication monitoring indicators. The correlation between the practice-level random effects for monitoring and prescribing indicators was -0.056 .

\subsection{Importance of Secondary Care Data in SIR}

The univariate models were re-fitted to the SIR data excluding secondary care data. Figures 2 and 3 show that using linked primary and secondary care data compared with using primary care data alone only affects the results for the monitoring indicators. This is mainly because some of the test results associated with monitoring indicators appear only in secondary care data.

\section{Discussion}

\subsection{Main Results}

We considered 18 prescribing and four monitoring medication safety indicators. For prescribing indicators, increasing age and polypharmacy increased the odds of 


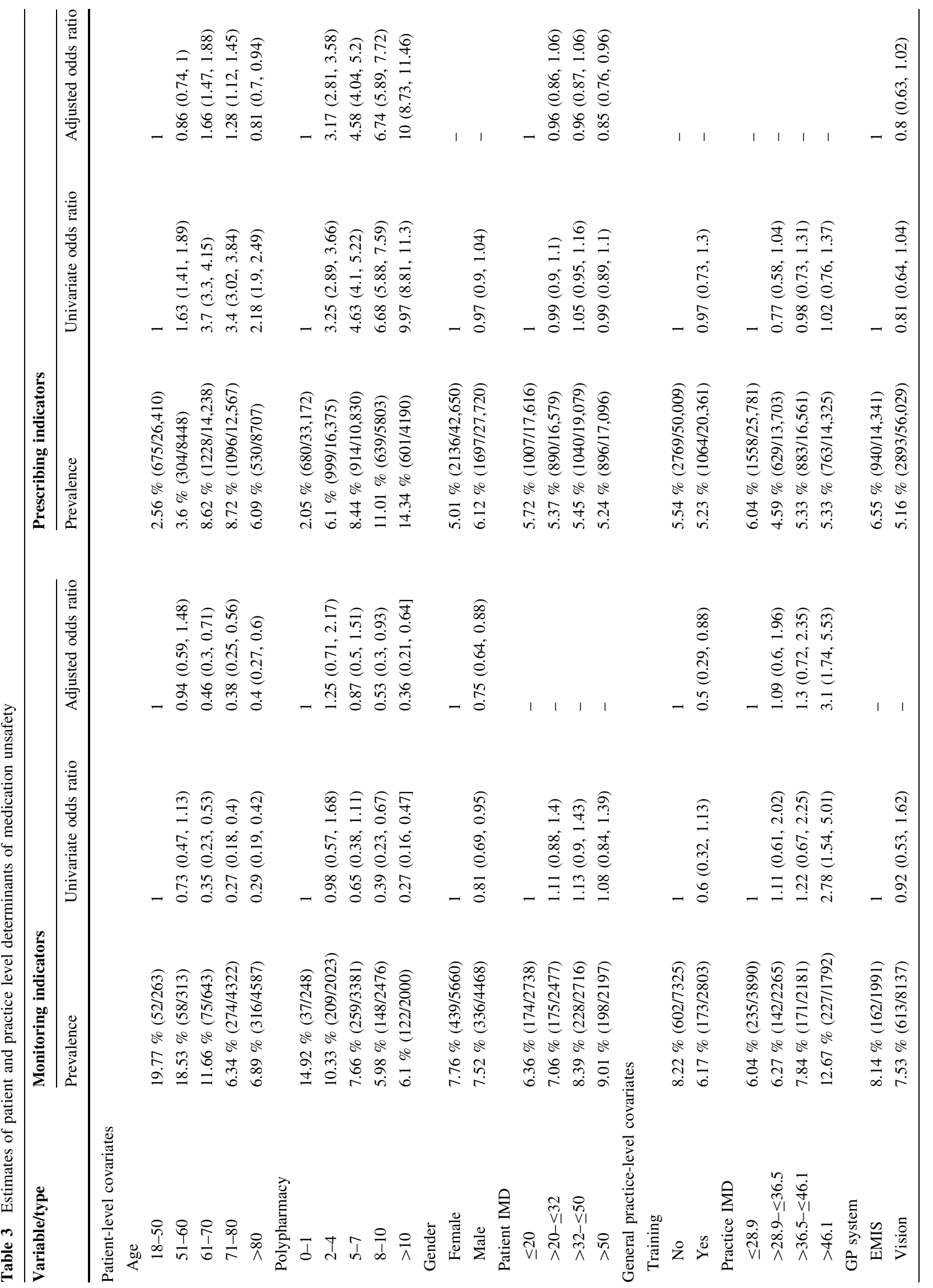


experiencing a prescribing hazard. Similar results were reported by previous studies $[3,5,6]$. Although polypharmacy is the most important risk factor for prescribing hazards, it should be noted that patients' co-morbidities, previous medication therapies (especially unsuccessful ones), treatment goals, and current health status were not taken into account. These are important contextual factors for analysing prescribing errors [23]; however, with the exception of co-morbidities, these factors are not readily accessible or quantifiable. Some apparent prescribing errors may be the only viable or necessary course of medication therapy in certain cases. This is especially relevant to elderly patients as the clinical trials underpinning many drug therapies exclude frail and elderly patients, thus, creating significant uncertainty around the effects of pharmacotherapy in elderly patients [23, 24].

Monitoring indicators are not usually discussed in their own right in the literature. Increasing age, polypharmacy, and being male decreased the likelihood of missed monitoring. The effect of a practice being a training practice was significant only in the adjusted model; patients in such practices were less likely to miss monitoring. Also, practices in the most deprived areas exhibited much higher levels of missed monitoring. This issue warrants further research to trace the origins of the inequity, through deeper, quantitative and qualitative study of patient- and practicelevel determinants.

The observed effects for age and polypharmacy, better monitoring and worse prescribing rates, are not surprising. Given this, we would expect that the increased morbidity burden for the elderly leads to more consultations and higher levels of polypharmacy. Within this context, it may be more relevant to apply prescribing quality standards for the elderly/high polypharmacy groups, as these groups receive numerous interventions and are, on average, exposed to more risks.

The PINCER study [8] reported high prevalence values for several monitoring indicators, namely, M2 (blood/liver function test for patients on methotrexate) 35-36\% and M4 (thyroid function test for patients on amiodarone) $46 \%$. In our study, M2 was $6.74 \%$ and M4 was $38.01 \%$. The large difference in the prevalence values of M2 could be explained by additional test data captured by secondary care data in SIR. However, the question that remains unanswered is whether these additional test data were originally requested by practices and simply did not make it into primary care records or whether they were recorded as a result of patients visiting the hospital. The latter seems to be more likely as both the blood and the renal function tests are common tests in the hospital, whereas the thyroid function test is not as common and, hence, the smaller difference in prevalence values of M4. Unless the tests are directly related to the cause of attending the hospital, they 
Fig. 1 Log-odds of prescribing and monitoring indicators by practice

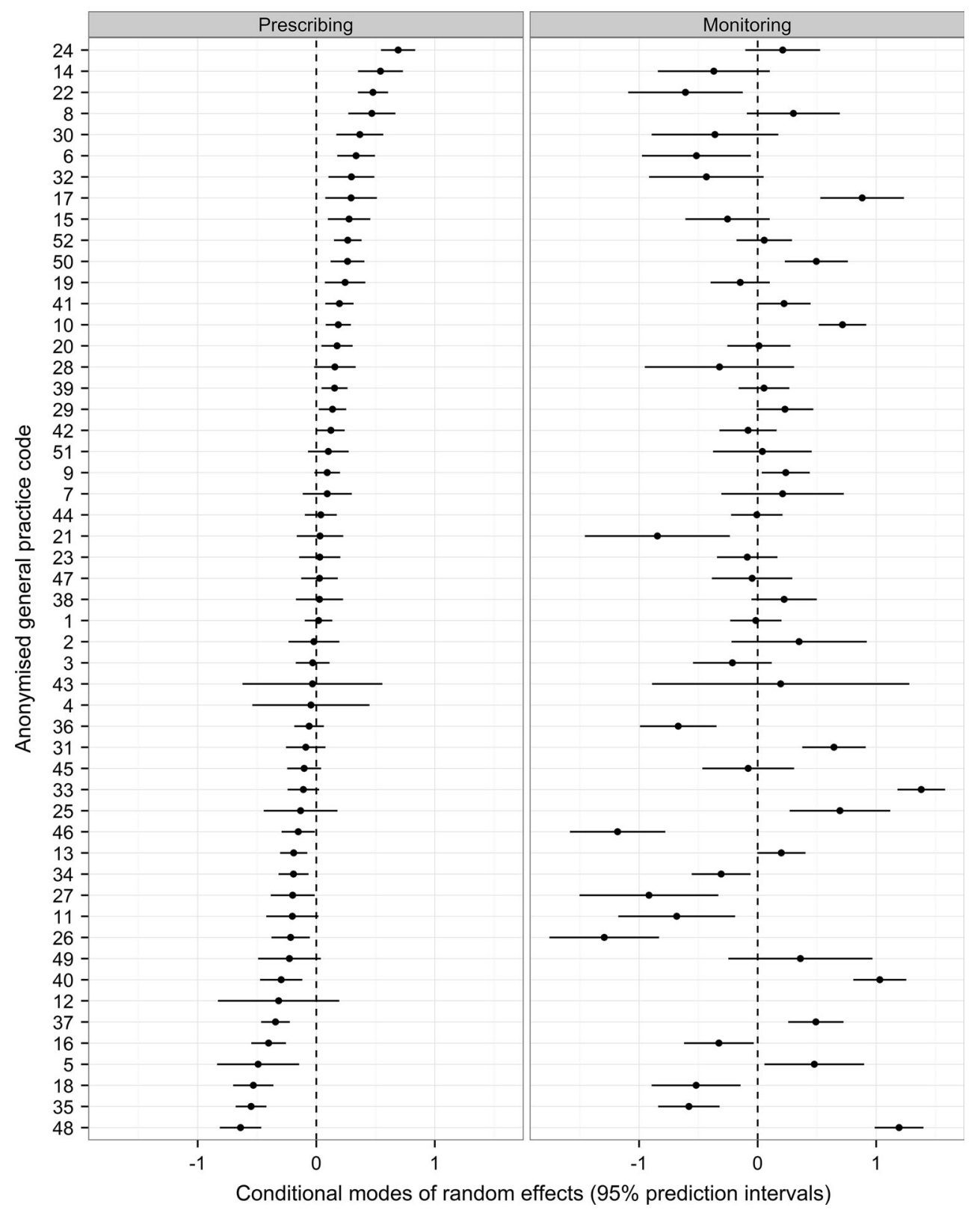

are unlikely to be communicated to a practice, although general practitioners in Salford can access the hospital results, if required. The fragmentation of monitoring medication-related biomarkers needs further investigation especially as there might be some missed opportunities when tests done in secondary care are not evaluated against a patient's long-term medication. The results of this study related to monitoring indicators require further investigation and should be interpreted with caution.

Analysis using only primary care data in SIR showed that the univariate effect of age for monitoring indicators was no longer significant. This might be due to excluding secondary care test data resulting from more frequent hospital visits by older patients. Polypharmacy was still a significant factor for monitoring indicators. As polypharmacy exhibits opposite effects for monitoring and prescribing indicators, it is clear that combining the two would dilute its effect. Also, the univariate effect of software system became significant, where Vision-derived data seemed to indicate higher levels of missed monitoring. This might be explained by the differences in data linkage between practice software systems and the hospital's information system. However, differences in performance across systems are not unprecedented and can be driven by 


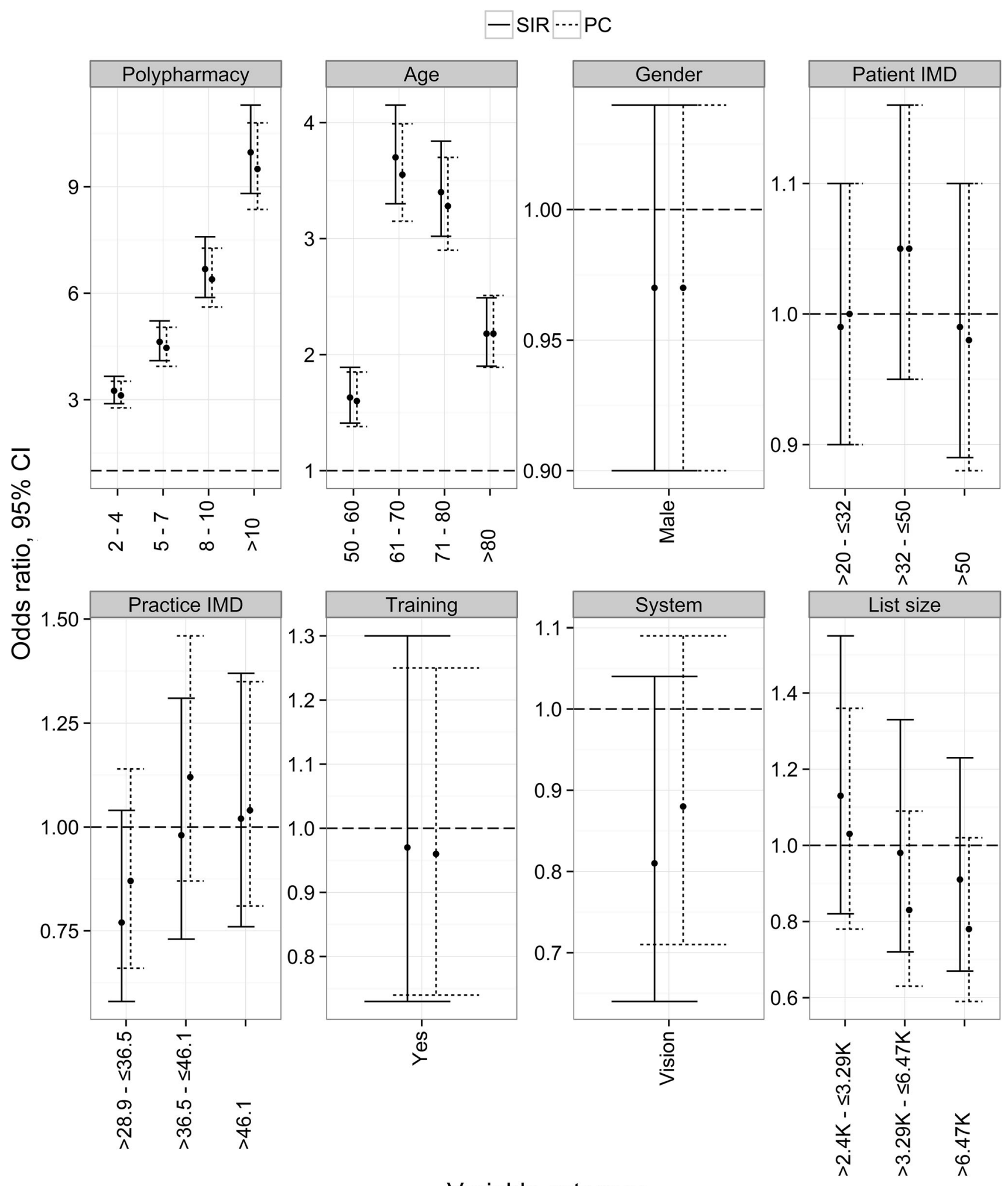

Variable category

Fig. 2 Univariate odds ratios for prescribing indicators by patient and practice factors. $C I$ confidence interval, $I M D$ Index of Multiple Deprivation, $P C$ primary care subset of SIR, SIR Salford Integrated Record (an electronic health record across primary and secondary care) 


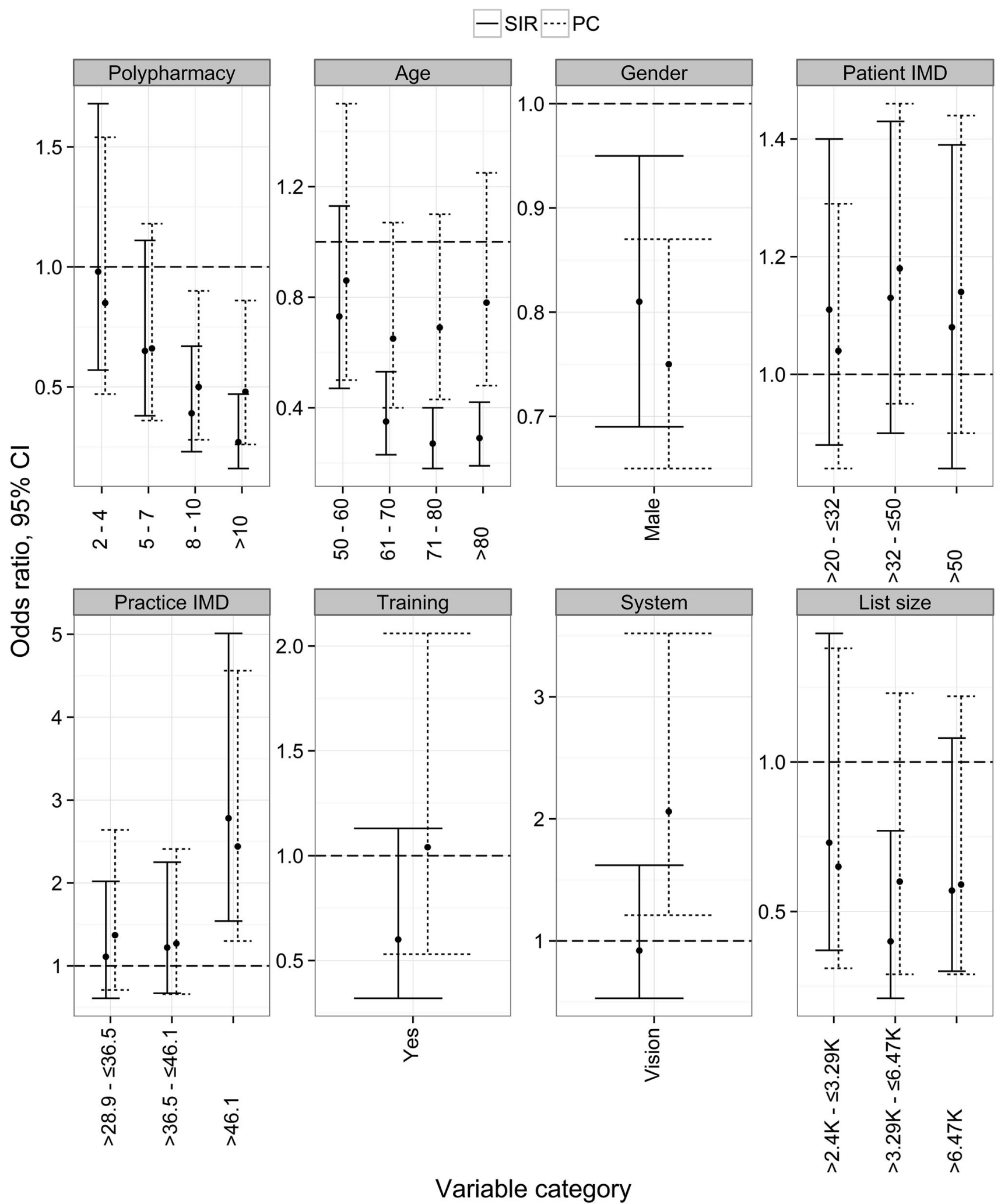

Fig. 3 Univariate odds ratios for medication monitoring indicators by patient and practice factors. $C I$ confidence interval, IMD Index of Multiple Deprivation, $P C$ primary care subset of SIR, SIR Salford
Integrated Record (an electronic health record across primary and secondary care) 
usability and intuitiveness, use of alerts and notifications, dismissability of reminders, support and training, or adaptability [25].

Adjusting for covariates reduced the variation between practices by 14 and $32 \%$ for prescribing and monitoring indicators, respectively. Clearly, there are other factors that affect the apparent differences in performance between practices. Also, practices with high numbers of prescribing safety issues do not necessarily have high numbers of monitoring issues. This indicates that the procedural and clinical context of medication prescribing and monitoring are different and the two processes should be investigated separately.

\subsection{Strengths and Limitations of the Study}

The main strength of this study is the comprehensive analysis of medication monitoring as well as prescribing safety indicators for a well-researched set of indicators and a defined population with linked primary and secondary care records. We have shown that linked secondary care data are important for medication safety surveillance in primary care. In addition, we have demonstrated the different statistical characteristics of monitoring compared with prescribing indicators, and shown how surveillance systems might deal with this.

One of the main limitations of this study is its crosssectional nature. Although we have provided some results using alternative reference dates (please see electronic supplementary material), more detailed longitudinal studies could be pursued. The variation in the outcomes within individual patients could change over time as concordance with medication policies change - a highly complex longitudinal picture.

There are also other important covariates that were not considered in this study, such as practitioner-level covariates like clinical experience. The extract of SIR we had access to did not contain practitioner-level data. In addition, a more complete picture of co-morbidity could be studied alongside polypharmacy, attempting to identify more detailed targets for quality improvement and, potentially, clinical decision-support systems.

Polypharmacy as a measure of medication exposure can be difficult to quantify, especially from integrated data such as that in SIR, where dosage and intake instructions need to be inferred through text mining. This requires further work, especially as a more accurate medication exposure measure would need to take into account whether drugs that have been prescribed are actually taken by patients, which could vary significantly depending on the type of medication.

We used a particular set of prescribing indicators which is not exhaustive of the safety concerns in prescribing and monitoring practice. Some of the indicators we investigated related to very small patient groups; hence, it is unlikely these indicators on their own will be adequate in quantifying practice prescribing safety. However, they should be adequate for this task as part of a larger indicator group.

The NHS is by far the largest health provider in the UK, although, alternative private providers do exist. The data we analysed only pertained to the NHS, and our findings are not necessarily generalisable to private health providers.

Although one of the ultimate aims of this study is to improve medication safety through computerised intervention, it should be noted that this is a two-way process. A computerised intervention with a feedback loop should aim at finding the contextual circumstances that lead to medication errors and not aim at providing punitive/summative assessments of practitioners' medication practice. In the context of manageable two-way interventions, the initial set of indicators is likely to be restricted. Having a large number of indicators can run the risk of over-whelming general practitioners with alerts and lead to "alert fatigue" where practitioners may override the alerts or stop providing feedback [26].

\subsection{Future Work}

As a follow-up to the current study, we are currently planning to roll out and evaluate an electronic audit and feedback intervention to improve medication safety in Salford primary care. The intervention, based on the PINCER trial [8], will interrogate linked electronic health record data to continuously assess the prescribing and monitoring indicators studied here. Each participating general practice will receive access to their own safety scores through a web-based dashboard application and will be visited by a trained pharmacist to review the indicator scores and initiate appropriate action. The results of this intervention study are expected by late 2016 .

\section{Conclusion}

Linked primary and secondary care records reveal important areas for quality improvement in health systems. Surveillance of prescribing and monitoring hazards ought to use linked primary and secondary care data. Surveillance systems built on integrated care record systems should be vigilant for inequities in age (older patients more often affected), examine polypharmacy (patients on multiple medications more often affected), treat prescribing and monitoring as different statistical processes (otherwise cross-contaminated effects will dilute potentially important signals), not assume that practices with higher prescribing 
hazards have more missed monitoring (uncorrelated here), and be vigilant for socio-economic inequity in monitoring (more missed monitoring in deprived areas).

Acknowledgments Permission to use the SIR data for the analysis was granted via the SIR approval board, which incorporates all the appropriate information governance. Further ethical approval was not required, because of the anonymised nature of the data. We would like to thank the SIR board for providing the SIR data for the analysis.

\section{Compliance with Ethical Standards}

Funding This work was supported by the University of Manchester's Health eResearch Centre (HeRC), funded by the Medical Research Council (MRC) Grant MR/K006665/1, and by the National Institute for Health Research Greater Manchester Primary Care Patient Safety Translational Research Centre (NIHR GM PSTRC). The views expressed are those of the authors and not necessarily those of the MRC, NHS, the NIHR, or the Department of Health.

Conflict of interest Artur Akbarov, Evangelos Kontopantelis, Matthew Sperrin, Susan J. Stocks, Richard Williams, Sarah Rodgers, Anthony Avery, Iain Buchan, and Darren M. Ashcroft have no conflicts of interest that are directly relevant to the content of this study.

Open Access This article is distributed under the terms of the Creative Commons Attribution-NonCommercial 4.0 International License (http://creativecommons.org/licenses/by-nc/4.0/), which permits any noncommercial use, distribution, and reproduction in any medium, provided you give appropriate credit to the original author(s) and the source, provide a link to the Creative Commons license, and indicate if changes were made.

\section{References}

1. Winterstein AG, Sauer BC, Hepler CD, Poole C. Preventable drug-related hospital admissions. Ann Pharmacother. 2002;36:1238-48.

2. Howard RL, Avery AJ, Slavenburg S, Royal S, Pipe G, Lucassen $\mathrm{P}$, et al. Which drugs cause preventable admissions to hospital? A systematic review. Br J Clin Pharmacol. 2007;63:136-47.

3. Guthrie B, McCowan C, Davey P, Simpson CR, Dreischulte T, Barnett K. High risk prescribing in primary care patients particularly vulnerable to adverse drug events: cross sectional population database analysis in Scottish general practice. BMJ. 2011;342:d3514.

4. Galvin R, Moriarty F, Cousins G, Cahir C, Motterlini N, Bradley $\mathrm{M}$, et al. Prevalence of potentially inappropriate prescribing and prescribing omissions in older Irish adults: findings from The Irish LongituDinal Study on Ageing study (TILDA). Eur J Clin Pharmacol. 2014;70:599-606.

5. Schubert I, Küpper-Nybelen J, Ihle P, Thürmann P. Prescribing potentially inappropriate medication (PIM) in Germany's elderly as indicated by the PRISCUS list. An analysis based on regional claims data: prescribing potentially inappropriate medication. Pharmacoepidemiol Drug Saf. 2013;22:719-27.

6. Holmes HM, Luo R, Kuo Y-F, Baillargeon J, Goodwin JS. Association of potentially inappropriate medication use with patient and prescriber characteristics in Medicare Part D: Inappropriate Medications and Part D. Pharmacoepidemiol Drug Saf. 2013;22:728-34
7. Morris CJ. Indicators for preventable drug related morbidity: application in primary care. Qual Saf Health Care. 2004; $13: 181-5$.

8. Avery AJ, Rodgers S, Cantrill JA, Armstrong S, Cresswell K, Eden $\mathrm{M}$, et al. A pharmacist-led information technology intervention for medication errors (PINCER): a multicentre, cluster randomised, controlled trial and cost-effectiveness analysis. Lancet. 2012;379:1310-9.

9. 2011 Census datasets: QS102EW Population density [Internet]. Office for National Statistics; Available from: http://www.ons. gov.uk/ons/index.html. Accessed 1 May 2015.

10. Avery AJ, Dex GM, Mulvaney C, Serumaga B, Spencer R, Lester HE, et al. Development of prescribing-safety indicators for GPs using the RAND Appropriateness Method. Br J Gen Pract. 2011;61:e526-36.

11. Spencer R, Bell B, Avery AJ, Gookey G, Campbell SM. Identification of an updated set of prescribing-safety indicators for GPs. Br J Gen Pract. 2014;64:e181-90.

12. Beers MH, Ouslander JG, Rollingher I, Reuben DB, Brooks J, Beck JC. Explicit criteria for determining inappropriate medication use in nursing home residents. UCLA Division of Geriatric Medicine. Arch Int Med. 1991;151:1825-32.

13. Wenger NS, Shekelle PG. Assessing care of vulnerable elders: ACOVE project overview. Ann Int Med. 2001;135:642-6.

14. Gallagher P, Ryan C, Byrne S, Kennedy J, O'Mahony D. STOPP (Screening Tool of Older Person's Prescriptions) and START (Screening Tool to Alert doctors to Right Treatment). Consensus validation. Int J Clin Pharmacol Ther. 2008;46:72-83.

15. Gallagher P, O'Mahony D. STOPP (Screening Tool of Older Persons' potentially inappropriate Prescriptions): application to acutely ill elderly patients and comparison with Beers' criteria. Age Ageing. 2008;37:673-9.

16. Communities and Local Government. The English indices of deprivation 2010: technical report. 2011.

17. R Core Team. R: A language and environment for statistical computing [Internet]. Vienna: $\mathrm{R}$ Foundation for Statistical Computing; 2014. Available from: http://www.R-project.org/. Accessed 12 Jan 2015.

18. Bates D, Maechler M, Bolker B, Walker S. lme4: Linear mixedeffects models using Eigen and S4 [Internet]. R package version 1.0-5; 2013. Available from: http://CRAN.R-project.org/ package=lme4. Accessed 12 Jan 2015.

19. Akaike H. A new look at the statistical model identification. Autom Control IEEE Trans On. 1974;19:716-23.

20. Nakagawa S, Schielzeth H. A general and simple method for obtaining R2 from generalized linear mixed-effects models. Methods Ecol Evol. 2013;4:133-42.

21. Lesnoff, M. aod: Analysis of Overdispersed Data [Internet]. R package version $1.3 ; 2012$. Available from: http://cran.r-project. org/package=aod. Accessed 12 Jan 2015.

22. Wickham H. ggplot2: elegant graphics for data analysis. New York: Springer; 2009.

23. O'Connor MN, Gallagher P, O'Mahony D. Inappropriate prescribing. Drugs Aging. 2012;29:437-52.

24. Penge J, Crome P. Appropriate prescribing in older people. Rev Clin Gerontol. 2014;24:58-77.

25. Kontopantelis E, Buchan I, Reeves D, Checkland K, Doran T. Relationship between quality of care and choice of clinical computing system: retrospective analysis of family practice performance under the UK's quality and outcomes framework. BMJ Open. 2013;3:e003190.

26. Nanji KC, Slight SP, Seger DL, Cho I, Fiskio JM, Redden LM, et al. Overrides of medication-related clinical decision support alerts in outpatients. J Am Med Inform Assoc. 2014;21:487-91. 